\title{
The Reddest Quasars: A Transitional Phase in Quasar/Galaxy Co-Evolution
}

\author{
Eilat Glikman* \\ Yale \\ E-mail: eilat.glikman@yale.edu
}

Quasars are extremely luminous sources powered by accretion of gas onto a supermassive black hole in the nucleus of some galaxies. Most of the $>10^{5}$ quasars identified in the literature have been identified in optical surveys through the "ultraviolet excess" (UVX) method. However, these samples are known to be incomplete and biased because of obscuration and anisotropic radiation. To overcome some of these biases and search for candidate obscured quasars, we matched radio sources from the FIRST $1.4 \mathrm{GHz}$ survey with the 2MASS near-infrared survey and selected objects with red optical-to-near-infrared colours. We followed up our candidates with optical and/or near-infrared spectroscopy and identified 119 dust-reddened quasars, defined as having at least one broad emission line in and a reddening of $E(B-V)>0.1$. The sample spans a wide redshift range, $0.1<z<3$ and reaches a reddening, $E(B-V) \lesssim 1.5$. When corrected for extinction, red quasars are the most luminous objects at every redshift and the fraction of red quasars increases with luminosity. The properties of red quasars suggest that they are revealing an evolutionary phase where the heavily obscured quasar is emerging from its dusty environment prior to becoming a "normal" blue quasar. We compute the fraction of quasars that are in this red phase and determine that its duration is $\sim 20 \%$ as long as the unobscured quasars phase: a few million years.

Frank N. Bash Symposium New Horizons In Astronomy, October 9-11, 2011

Austin Texas

\footnotetext{
*Speaker.
} 


\section{Introduction}

A fundamental goal of observational cosmology is to answer the question of how galaxies evolved throughout cosmic time. Several pieces of observational evidence suggest that the growth of supermassive black holes (SMBHs) may play a key role in answering this question. Locally, SMBHs are a ubiquitous feature in massive galaxies [1] and their masses appear to correlate with the global properties of their host galaxies, such as the stellar velocity dispersion (the $M_{\mathrm{BH}}-\sigma$ relation [2,3]) and the luminosity of the stellar bulge [4]. A recent compilation of the $M_{\mathrm{BH}}-\sigma$ relation is shown in Figure 1, left [5].

These observations are remarkable: stars in the bulge, located kiloparsecs away from their galactic centers, are not influenced by the gravitational potential of the $\mathrm{SMBH}$, whose sphere of influence is a few parsecs. Since the stars cannot "know" about the SMBH, perhaps they arrived on this relation in tandem with the $\mathrm{BH}$.

A third piece of evidence that supports the assertion that SMBHs and their host galaxies "coevolved" is seen by examining the cosmological evolution of BH growth and star-formation rate. We see in Figure 1, right, that the quasar luminosity density (a proxy for the growth of SMBHs via accretion) rises sharply with redshift and peaks at $z \sim 2$ declining thereafter to $z \sim 6$. This behavior is mirrored in the evolution of the star formation rate density, suggesting that SMBHs and galaxies have built up symbiotically $[6,7,8]$.
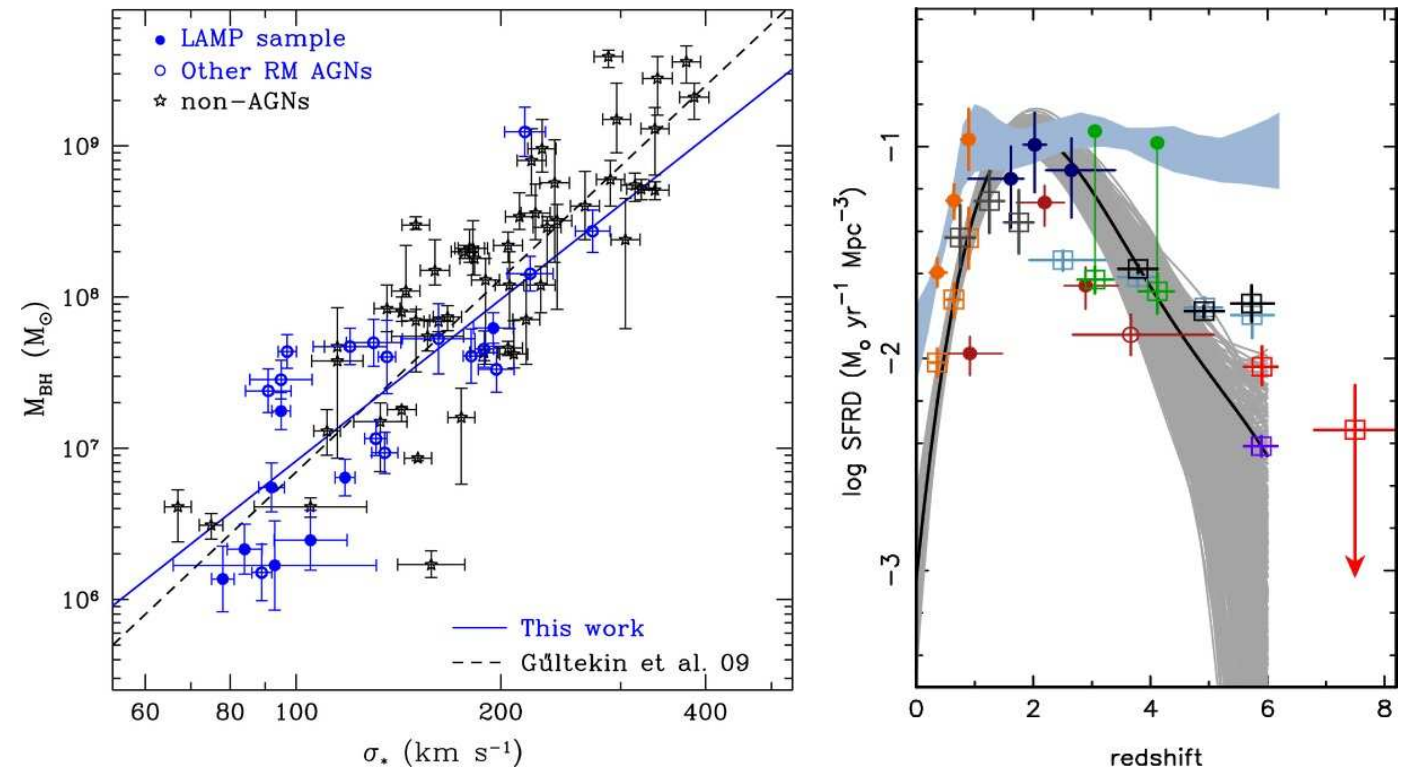

Figure 1: Left - The $M_{\mathrm{BH}}-\sigma$ relation from [5] showing the tight correlation between the mass of the SMBH of a galaxy and the stellar velocity dispersion, spanning $\sim 4$ orders of magnitude in BH mass. Right - The redshift evolution of QSO luminosity density is plotted in the shaded region over-plotted by measurements of the star formation rate [8].

Galaxy mergers appear to be very important in explaining the co-evolution of galaxies and SMBHs. Gas rich mergers produce bursts of star formation and provide fuel to feed the SMBH and power a quasar. The energy released by the quasar expels enough gas to quench both star formation and further black hole growth (i.e., feedback). Theoretical models of merging galaxies 
appear to require a SMBH in order to match observations. In particular, the absence of SMBHs, and the feedback that they generate, would produce far too much star formation than is observed $[9,11,10]$.

This merger-driven model for SMBH/galaxy co-evolution links different observed types of galaxies as stages of evolution. Sanders et al. (1988) presented a picture that "... ultraluminous infrared galaxies (ULIRGs) represent the initial, dust-enshrouded stages of quasars. Once these nuclei shed their obscuring dust, allowing the AGN to visually dominate the decaying starburst, they become optically selected quasars" [9]. Local ULIRGs are predominantly seen in merging systems. Quasars live in bulge-dominated, massive ellipticals [12, 13, 14]. If the evolutionary scenario is correct, then there is a missing stage of evolution where the buried, growing BH emerges from the ULIRG as it evolves into a luminous quasar.

\subsection{What is a Quasar?}

Quasars are extremely luminous sources, powered by accretion of gas onto a supermassive black hole in the nucleus of a galaxy. They were originally discovered as optical counterparts to bright radio sources. The first quasar, $3 \mathrm{C} 273$, was cataloged as a bright radio source in the Third Cambridge (3C) radio catalog in 1963. It was coincident with an abnormally blue star with unidentifiable emission features. It was soon realized that these unusual lines were redshifted Balmer emission lines, placing this object at $z=0.158$, the highest redshift known at the time [15]. Another bright radio source, also with a blue stellar optical counterpart, 3C48, was then interpreted as having an even higher redshift of $z=0.367$ [16].

In the $H_{0}=100$ cosmology of 1963, the high redshift of 3C273 implied that it was at a distance of $500 \mathrm{Mpc}$. This distance, combined with its bright optical magnitude $(V=13)$ further implied that it was hundreds of times the luminosity of a typical galaxy concentrated in a region smaller than $1000 \mathrm{pc}$ in diameter. Spectroscopic follow-up of dozens of optical counterparts to point-like, bright radio sources from the $3 \mathrm{C}$ and $4 \mathrm{C}$ radio catalogs led to discoveries of more such objects with the same characteristics, named Quasi-Stellar Radio Souces (Quasars):

- Broad emission lines in their optical spectra.

- Narrow forbidden emission lines of high ionization atomic species.

- Large ultraviolet flux of radiation.

- Extremely large luminosities emanating from very compact regions.

- High redshifts.

- Flux variability in many cases.

Using the fact that quasars had blue $U-B$ colors and stellar appearances on optical photographic plates, a population of objects with optical properties identical to quasars was discovered, but without the radio emission ("radio quiet quasars") [17]. With improved color selection and high-quality, wide-field, optical surveys, there are now over $10^{5}$ known quasars and only $\sim 10 \%$ are radio-loud. Figure 2 shows a quasar composite spectrum spanning the optical through nearinfrared [18]. 


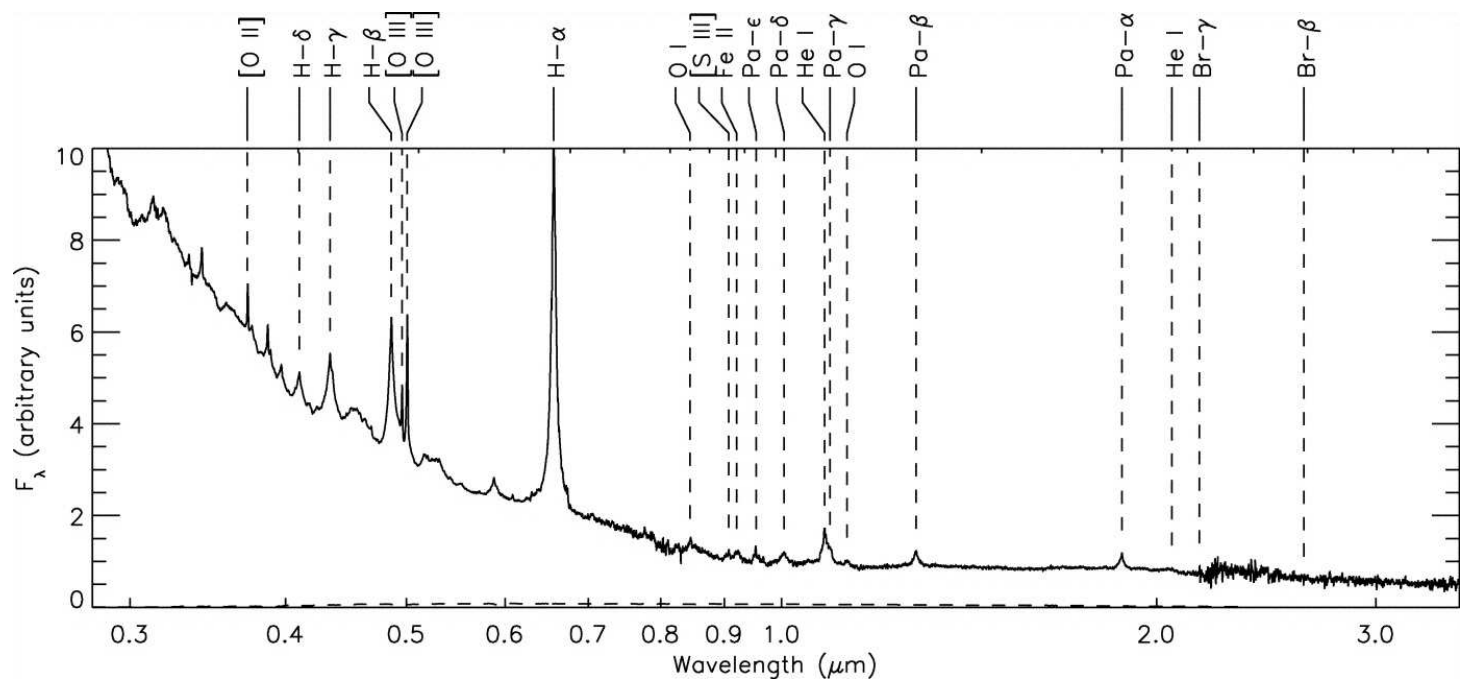

Figure 2: Optical through near-infrared composite spectrum from [18], demonstrating the strong blue optical and ultraviolet shape of the spectral energy distribution as well as the broad emission lines.

We now have a clearer picture of the physics of accretion onto nuclear SMBHs depicted by Active Galactic Nuclei (AGN), of which quasars are the most luminous type. These classifications are different manifestations of the same physical phenomena giving rise to a continuum in luminosity. Quasars outshine their host galaxies, appearing stellar in images, and can be seen to cosmological distances. They are rare, with a low volume density. On the other hand, AGN are lower luminosity. They are more numerous than quasars and are found at low redshifts in the nuclei of resolved galaxies.

\subsection{Obscuration and Redding in Quasars}

The properties of local AGN support an axisymmetric, orientation-based model for their structure. This model, shown in Figure 3, can explain many of their observed properties. In particular, the presence of absorption from dusty clouds, often modeled as a torus, outside the broad line region results in a spectrum showing only narrow emission lines (referred to as "Type II" sources). This model is also successful at unifying the observed properties of radio galaxies [19].

At low redshift, low-luminosity AGN fit the orientation-based picture very well. The Type I AGN are those objects observed from the pole direction. Type II AGN are those sources whose inner parts can only be seen through reflected radiation, from their polarized light. At high redshifts, Type II (obscured) quasars had been elusive. Large samples have been recovered in the Sloan Digital Sky Survey [37, SDSS] and subsequently in deep X-ray surveys [20, 21, 22, 23, 24] .

An important corollary to the orientation-based explanation is that the covering fraction of the obscuring clouds decreases with increasing luminosity; this is known as the receding torus model [25]. An increase in the ratio of obscured/unobscured AGN with decreasing luminosity has been seen in samples of X-ray selected AGN samples that show, in support of this model[26, 27, Figure 3 , right]. 

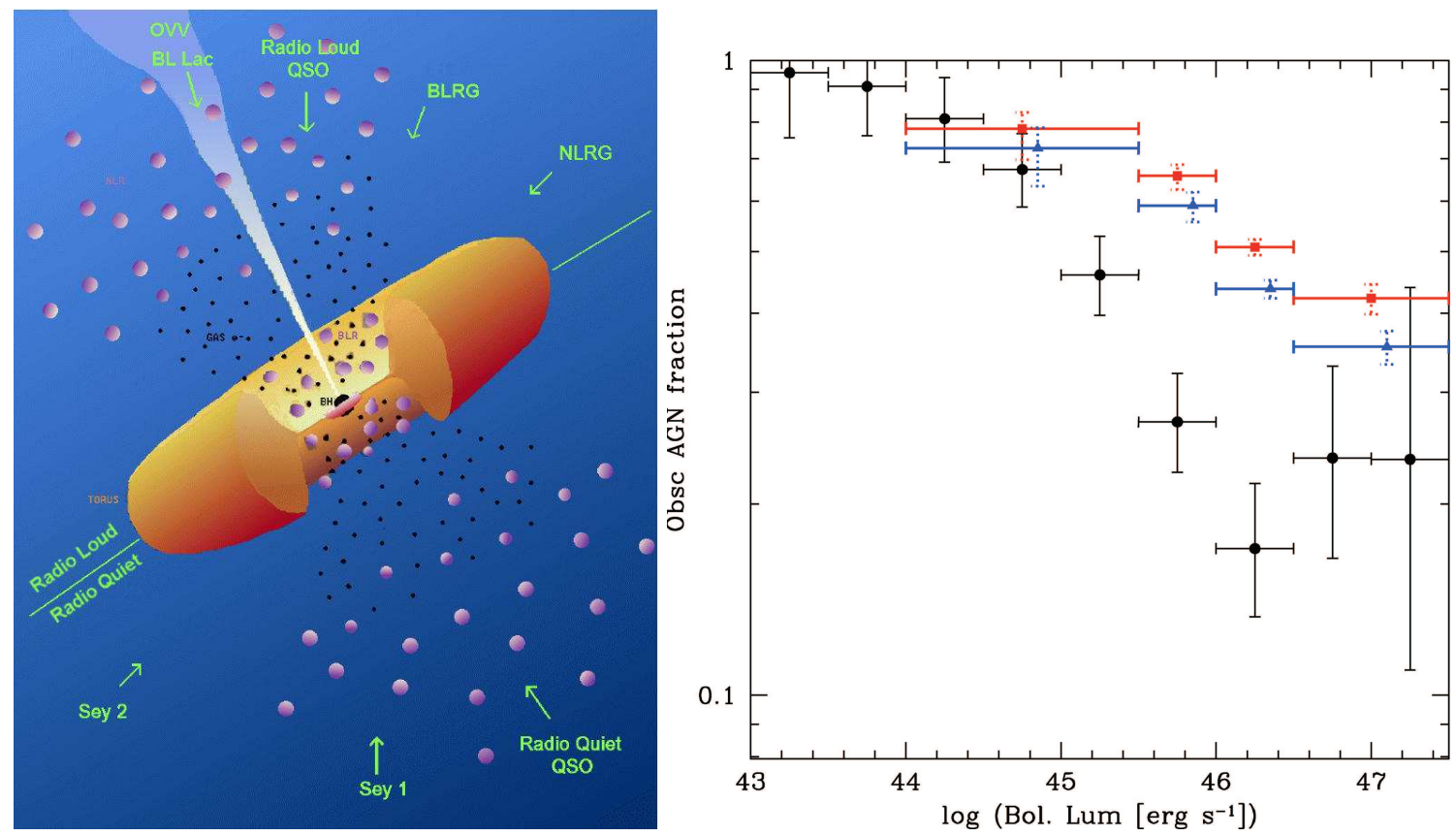

Figure 3: Left - Schematic unification model for AGN demonstrating the dependence of the observed radiation on the viewing angle [19]. Right - Fraction of obscured AGN as a function of AGN bolometric luminosity [27]. The fraction of obscured AGN increases strongly with decreasing luminosity, consistent with the receding torus model and AGN unificaiton.

\section{Finding Red Quasars: The FIRST-2MASS Survey}

The AGN obscuration described above is the result of orientation and is not part of the evolutionary picture outlined in the Introduction. To identify the missing link in the path from ULIRG to quasar, we must search for a new and different class of obscured quasars: red quasars. The rest of this paper concerns these objects, how they are different from other quasars, and why it matters.

There are $>10^{5}$ quasars identified in the literature. Most are found in the optical, in searches for blue, ultraviolet excess (UVX) objects. However, the presence of dust along the line of sight to a quasar will both redden and dim the light from the quasar. As a consequence, flux-limited optically-selected quasar samples are biased against dust-obscured objects. A few magnitudes of extinction will remove faint objects and leave only the most luminous sources in a flux-limited sample [28, Figure 4]. Quasars will also be missed because their colors no longer obey the UVX color cuts. In fact, reddening can cause quasars' colors to shift into the stellar locus in their optical colors making them nearly impossible to find in optical surveys.

To address this, we conducted a deliberate search for red quasars utilizing three criteria in three wavelength regimes. We matched the FIRST $^{1}$ radio catalog [29], which mapped a quarter of the sky at $1.4 \mathrm{GHz}$ to a sensitivity of $1 \mathrm{mJy}$, to the near-infrared 2MASS survey ${ }^{2}$ (referred to as the F2M survey, hereafter) and selected objects that were not detected in the Automated Plate Machine (APM) scans of the first generation Palomar Sky Survey (POSS-I). This effectively imposed optical

\footnotetext{
${ }^{1}$ Faint Images of the Radio Sky at Twenty Centimeters, http: / / sundog.st sci.edu

${ }^{2}$ Two-Micron All Sky Survey, http: //www.ipac.caltech.edu/2mass /
} 

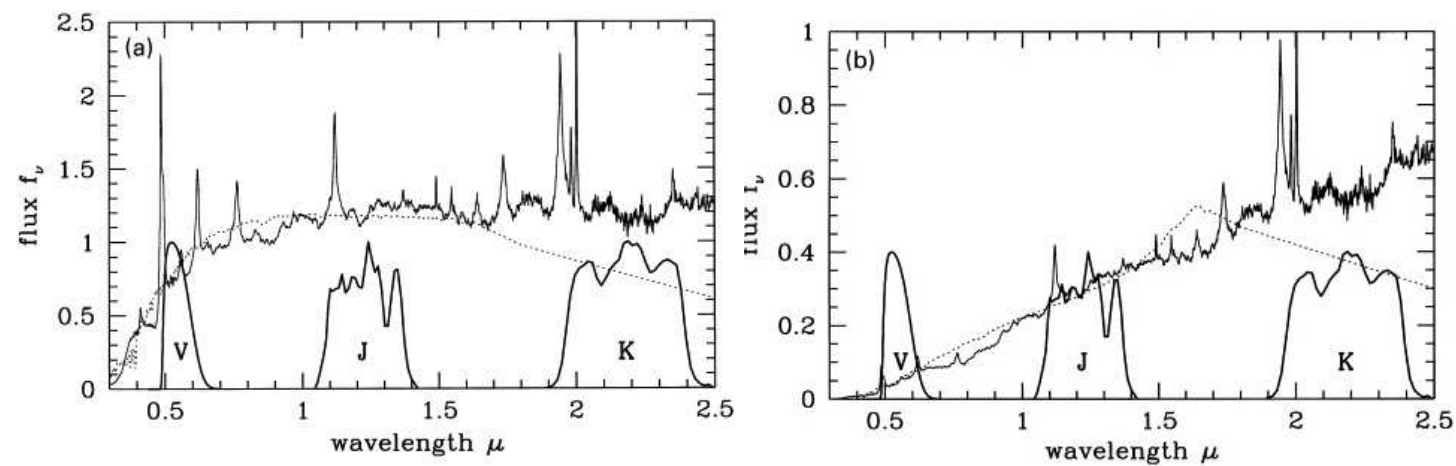

Figure 4: Optical through near-infrared spectral energy distribution for quasars (solid line) and stars (dotted line), showing typical optical and near-infrared bandpasses $V, J<$ and $K$. Left - An unreddened quasar at $z=3$ with a $\mathrm{K}$ star. Right - Reddened quasar with $\mathrm{E}(\mathrm{B}-\mathrm{V})=0.3$ at $\mathrm{z}=2.5$ and an early $\mathrm{M}$ star. While optical light is lost due to extinction, near-infrared light suffers little extinction and is a better wavelength for selecting reddened quasars [28].

flux limits of $R \gtrsim 20$ and $B \gtrsim 21.5$. We chose to include the radio criterion in order to avoid strong contamination from low mass stars (e.g., $M$ dwarfs) which are abundant in the near infrared, but do not emit strongly in the radio. This pilot project resulted in 69 candidates over $2716 \mathrm{deg}^{2}$. Spectroscopic identification of 54 of these resulted in a sample of 17 heavily reddened quasars [30]. Figure 5 shows the optical-to-near-infrared colors for this sample. We identified a set of color cuts, $R-K>4$ and $J-K>1.7$ which maximize our efficiency of finding red quasars while avoiding stars (yellow circles) and galaxies (green circles).
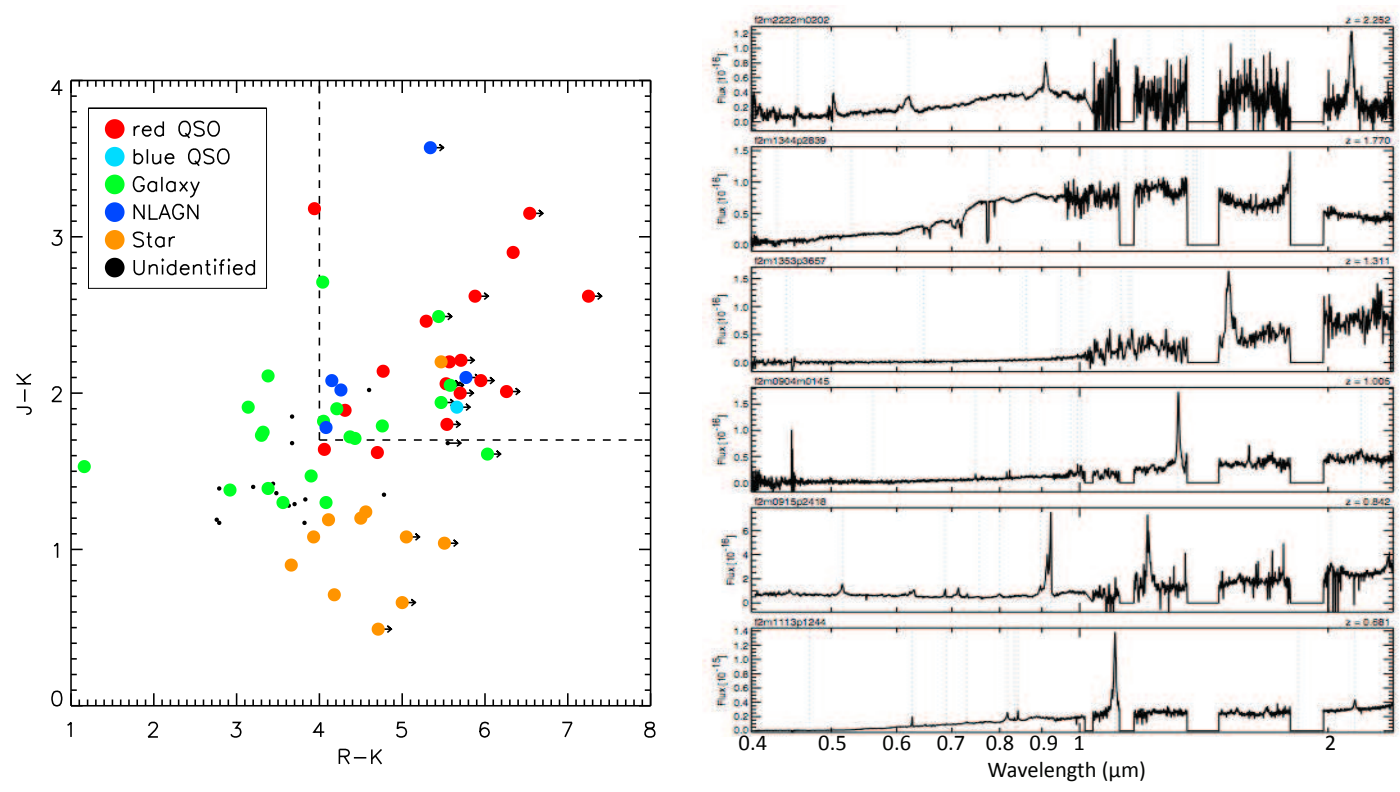

Figure 5: Left $-J-K$ vs. $R-K$ colors of optical drop outs in the FIRST-2MASS pilot study [30]. Spectroscopically identified objects are colored and labeled in the legend. The dashed lines define color cuts that maximize red quasar selection. Right - Example spectra from the F2M red quasar survey covering optical-to-near-infrared wavelengths $(0.4-2.4 \mu \mathrm{m})$. 
Having identified an efficient color selection method for finding red quasars, we expanded the survey to include objects with optical detections, requiring only that they obey the aforementioned color cuts [31, 32] and searched the full area of the FIRST survey [33]. This effort produced a catalog of 394 candidates over $9033 \mathrm{deg}^{2}$. We performed a spectroscopic followup campaign in the optical and near-infrared and produced 316 spectroscopic identifications (80\% completeness). Among these spectra, 119 are red quasars - by far the largest sample of dust reddened quasars, whose selection is uniform and well-understood, to date. A set of sample spectra from the F2M red quasar survey are shown in Figure 5, right.

\section{Properties of Red Quasars}

To determine the amount of dust along the line-of-sight to each quasar, we fit a reddened quasar template to our red quasar spectra applying an exponential reddening law to the composite.

$$
F=F_{0} \exp ^{-k(\lambda) E(B-V) / 1.086}
$$

Where $k(\lambda)$ is the Small Magellenic Cloud (SMC) reddening law [34]. Example fits are shown in Figure 6, left.

We de-redden the F2M red quasar spectra to study their intrinsic properties. With the intrinsic brightness of the F2M red quasars in hand we can compare their number counts to the number counts of blue, optically-selected quasars to determine the fraction of quasars that are missed in optical flux-limited surveys that rely on UVX (or other blue color) optical selection. In Figure 6 , right, we show the surface density of F2M red quasars before (red filled squares) and after (purple open squares) de-reddening. We compare their number counts per square degree with those of quasars from the FIRST Bright Quasar Survey ([35, 36] FBQS; crosses and x symbols). FBQS quasars were selected by matching the FIRST survey with the APM catalog and selecting objects with blue colors. We then match this sample to 2MASS to produce a quasar catalog that reaches the same flux depth as F2M in the radio and near-infrared, which makes it a natural blueequivalent survey to F2M. We also constructed a radio- and infrared-detected catalog from the SDSS by matching the fifth quasar catalog from SDSS [38] to FIRST and 2MASS (triangles).

Comparing the space densities of red and blue quasars, we find that the observed fraction of F2M quasars make up $10 \pm 1 \%$ in flux limited samples. In other words, $10 \%$ more quasars are recovered when changing to a near-infrared flux-limited survey from an optical survey such as POSS-I or SDSS. Once we correct the red quasars for extinction we see that intrinsically, F2M quasars make up $19.2 \pm 2.6 \%$ of radio-selected quasars with $K \leq 14.5$ magnitudes.

Figure 7 shows the de-reddened absolute $K$-band magnitude for the F2M red quasars colored by the amount of reddening that we computed for each source (yellow circles are mildly reddened, while redder circles are more heavily reddenend). We plot for comparison the FBQS quasars with small black points, for which we assume no extinction correction is necessary. This figure reveals a remarkable trend: at all redshifts, red quasars are the most luminous objects. This trend showing an increase of red quasar fraction with increasing luminosity goes in the opposite direction as the trend for Type I/II quasars, i.e., the receding torus model. We conclude that the F2M quasars are not reddened by nuclear obscuration but are rather an evolutionary phase. 
$\left\{2 m 1353+3657 \quad z=1.311 \mathrm{~A}(\mathrm{~B})=11.59^{\circ} \mathrm{A}(\mathrm{K})=1.49^{\circ} \mathrm{E}(\mathrm{B}-\mathrm{V})=1.13^{\mathrm{m}} \mathrm{ms}=21.43\right.$
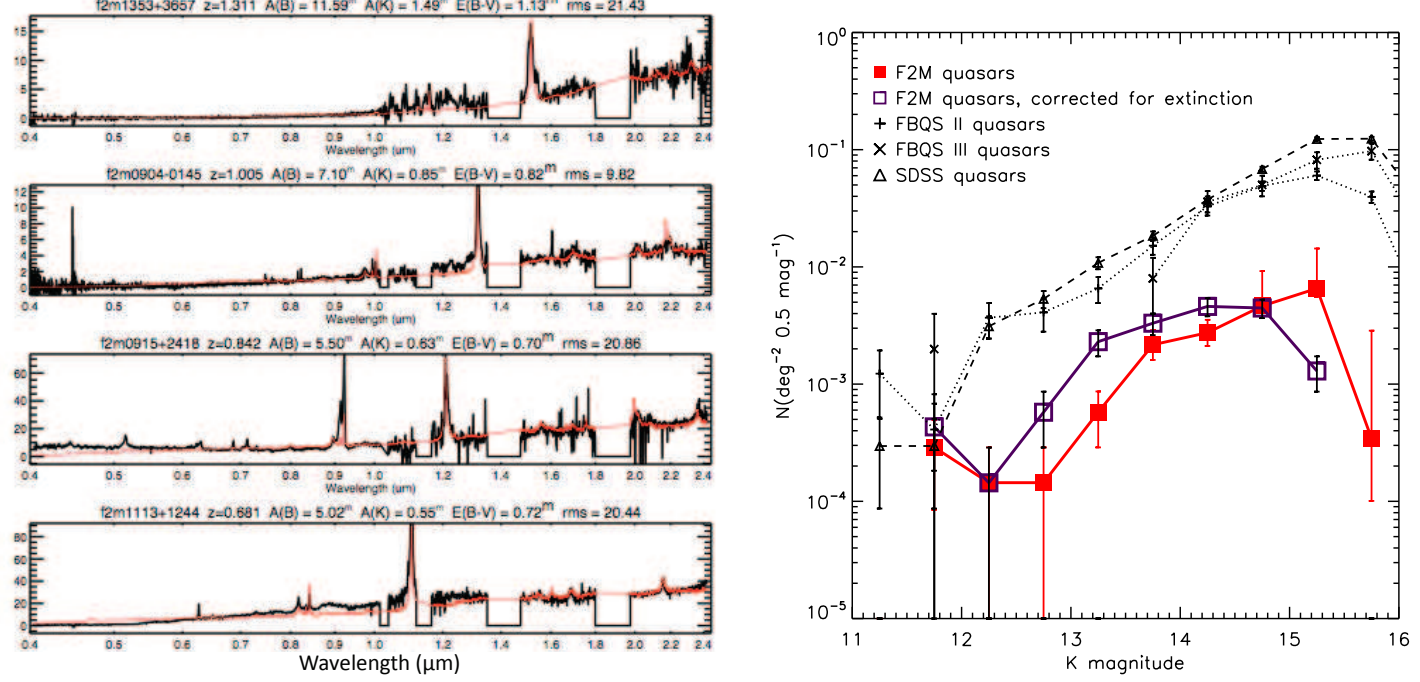

Figure 6: Left - Examples of fits of a reddened quasar template to F2M red quasars to determine $E(B-V)$, for each quasar which enables de-reddening. The quasar spectra are plotted with a black line and the reddend template fit is overplotted with a red line. Right - Number counts per square degree for F2M red quasars before and after de-reddening (filled red and open violet squares, respectively) compared with the same for FBQS (crosses and x's) and SDSS quasars matched to FIRST and 2MASS (triangles).
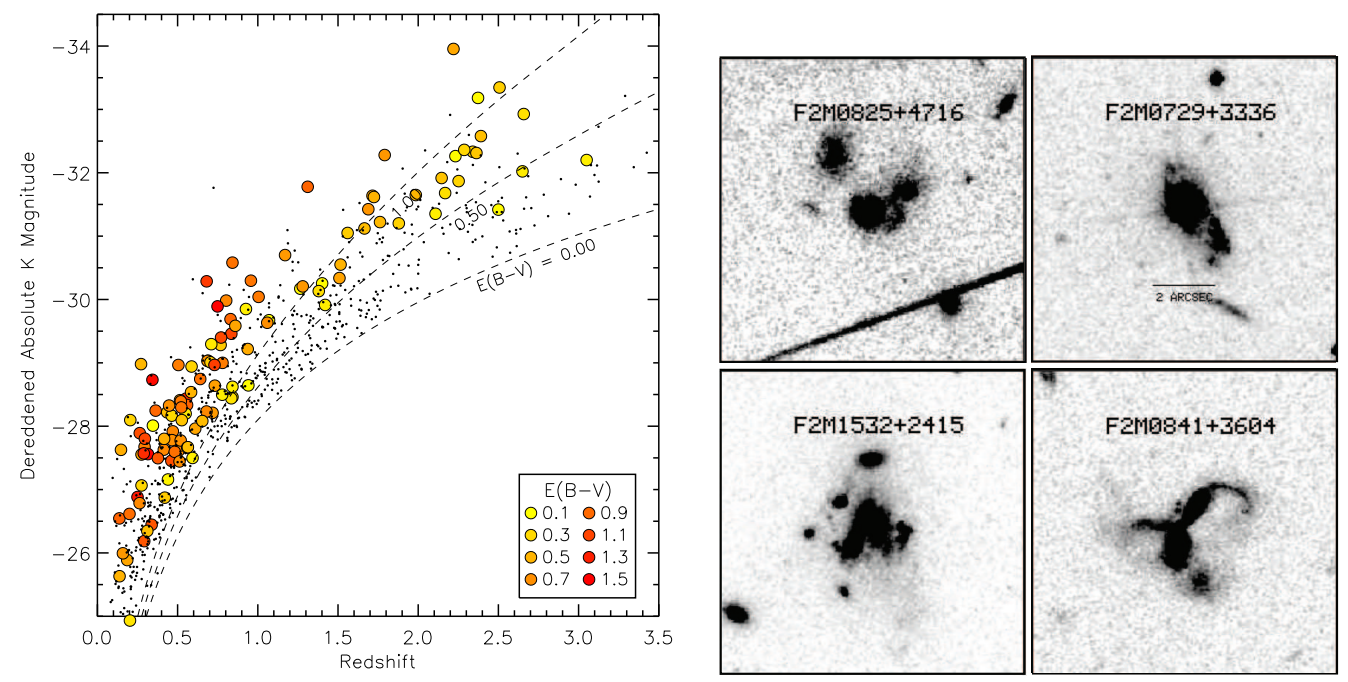

Figure 7: Left - De-reddened absolute $K$-band magnitude of red quasars (circles) colored by the amount of reddening that they experience, defined in the legend, compared with the same for FBQS quasars, which we assume are not obscured. At every redshift, red quasars are the most luminous objects. Right - Hubble Space Telescope images of four objects from our F2M sample showing intense merging and interaction in these reddened quasars. 

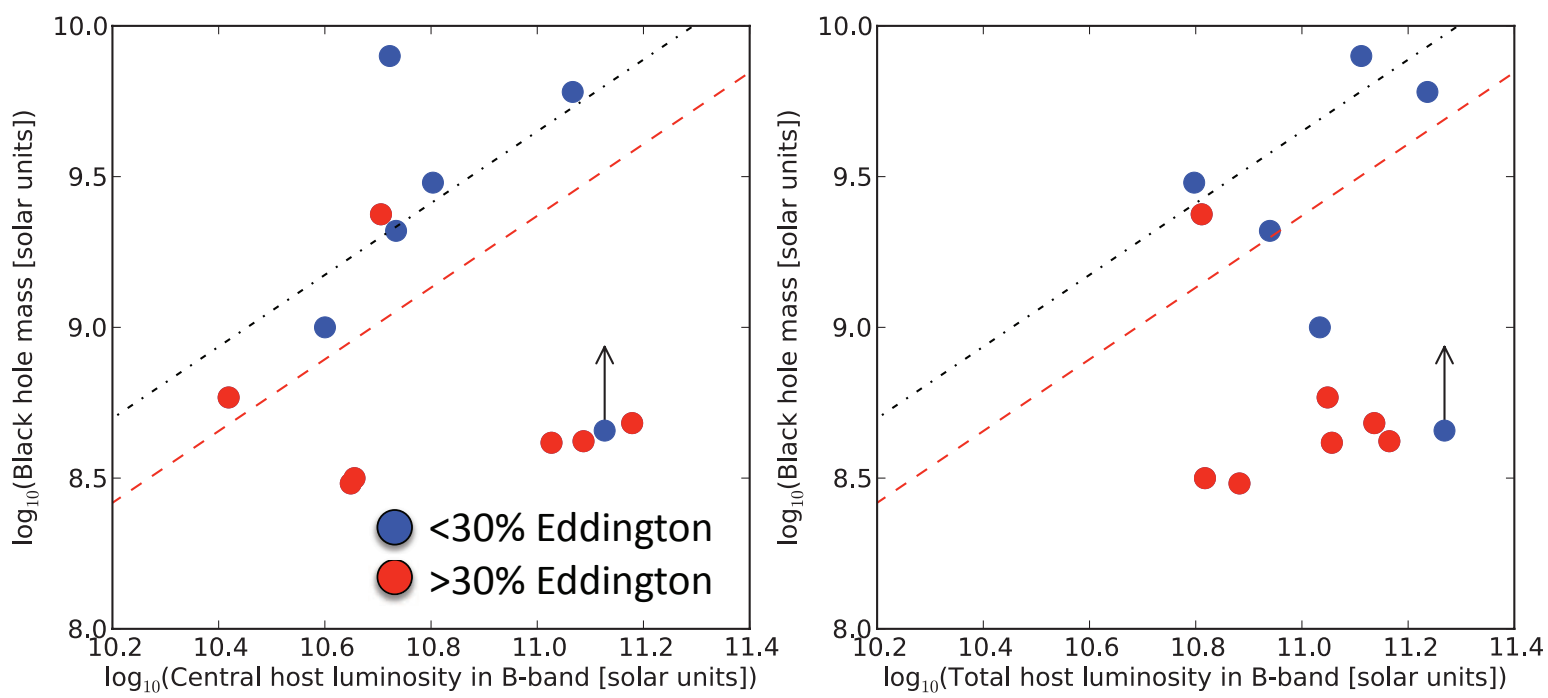

Figure 8: Black hole mass vs. host luminosity for the F2M red quasars [40]. Red circles are quasars with accretion rates higher than $30 \%$ of the Eddington rate. The objects with the highest accretion rates fall below the relation, suggesting that they are still growing their black holes while the stellar mass has already assembled.

\section{Red Quasars are a Phase in Quasar/Galaxy Co-Evolution}

We describe here the full body of evidence in support of red quasars being a phase in quasar/galaxy co-evolution rather than an orientation-based phenomenon. Thirteen red quasar hosts were imaged with the Advanced Camera for Surveys (ACS) on the Hubble Space Telescope (HST) during Cycle 13. All the images reveal a nearby companion and eleven show interacting or disrupted morphologies, as shown in Figure 7, right [39]. These images suggest that the F2M red quasars are a consequence of merger activity, as predicted by the merger driven quasar/galaxy co-evolution model.

We have also obtained Spitzer infrared IRS spectroscopy (covering $5-38 \mu \mathrm{m}$ ) and MIPS photometry $(24,70$, and $160 \mu \mathrm{m})$ of the same thirteen red quasars that had HST images. This long wavelength sampling of the quasars' spectral energy distribution (SED) allowed us to determine their bolometric luminosities. Incorporating this with a black hole mass estimate based on the width of the broad lines in the red quasar spectra, we compute their accretion rates and Eddington ratios. Figure 8 shows the $\mathrm{BH}$ mass vs. host luminosity relation for the F2M red quasars. We see a large fraction of objects with high black hole accretion rates (red circles have $L_{\mathrm{EDD}}>30 \%$ ), consistent with the idea that quasar accretion rates are high in this early phase of growth. Furthermore, the red quasars with the highest Eddington rates lie below the $M_{\mathrm{BH}}-L_{\mathrm{Bulge}}$ relation. This offset from the local relation is consistent with a picture in which some of the black holes are yet to grow to their equilibrium size following a major merger [40].

The existence of a population of red quasars is consistent with a merger-driven picture of $\mathrm{SMBH} /$ galaxy co-evolution in which gas rich galaxies, each hosting a $\mathrm{BH}$, merge and, in the pro- 
cess, induce both a starburst and fuel the growth of the nuclear SMBH. Figure 9 shows the predicted lightcurves from such a model [42]. On the left the full merger is shown over a 2 Gyr period. The light curves for the quasar phase, highlighted by a red oval in the lower left hand panel, is expanded on the right where the column density (i.e., obscuration) as a function of time is shown in the top panel, followed by the bolometric luminosity in the middle panel and the observed $B$-band luminosity in the bottom. In this model, the growing BH spends most of its time in a deeply buried, heavily obscured phase, followed by a clearing of the obscuring material via AGN feedback, revealing a blue, unobscured quasar as the final phase.

The characteristics of the F2M red quasars fit very well into this picture, not as the deeply obscured, earliest, growth phase (as has been seen in in ULIRGs [9]), but rather as a transitional phase from completely obscured to blue quasar. This transition is indicated by the vertical colored line in Figure 9, right. The fraction of red quasars therefore represents the duration of the phase, $\sim$ $20 \%$ of a typical quasar lifetime, which has been estimated to be a few $\times 10^{7}$ years [41], suggesting that the emergence phase lasts a few million years.
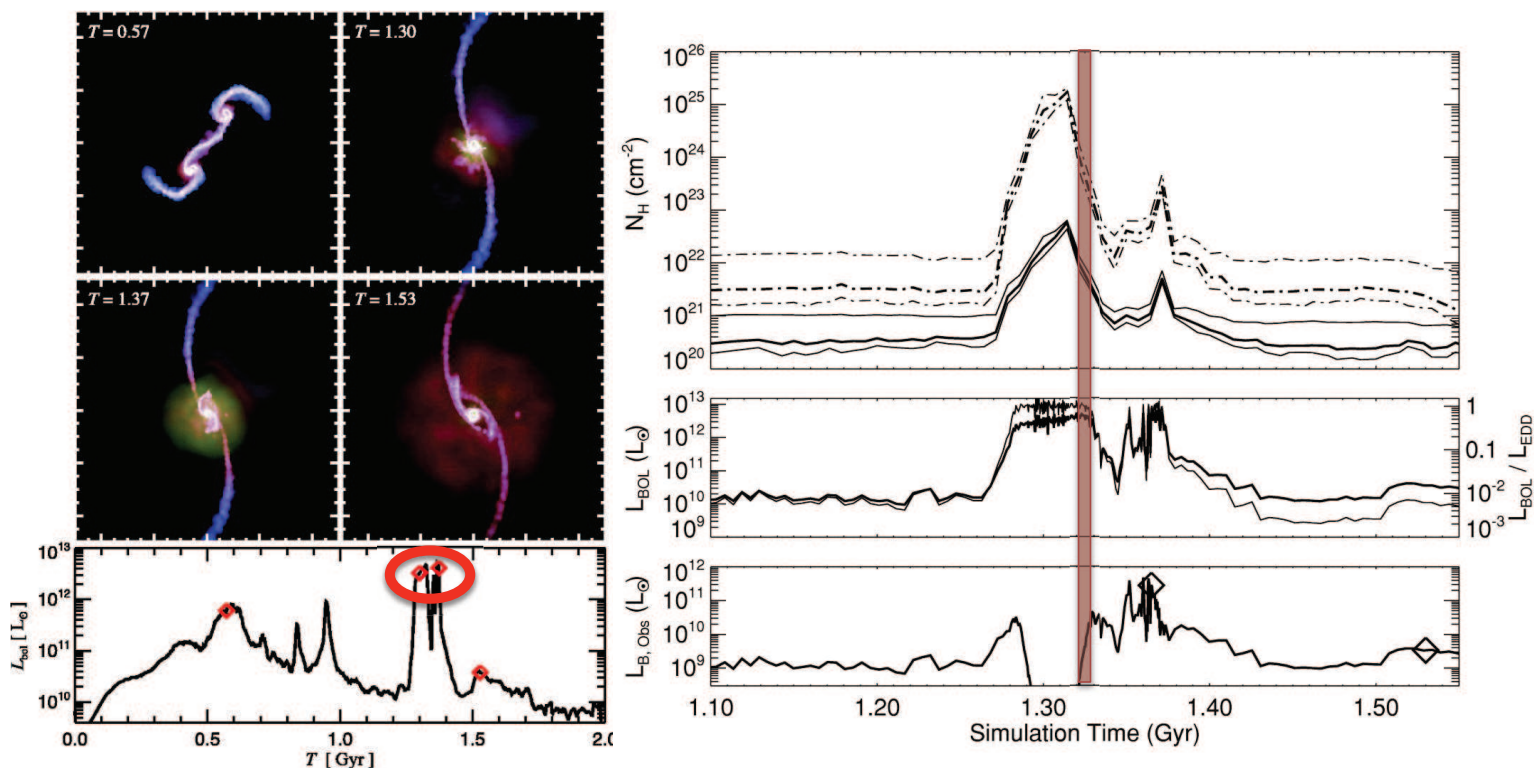

Figure 9: Quasar lightcurve during a major merger from [42]. Left - The full evolution of the bolometric luminosity over a 2 Gyr timespan. The quasar phase is highlighted with a red oval and zoomed in on the right-hand panel. Right - The evolution of the quasar phase of a major merger is shown for three observables. The the column density as a function of time is shown in the top panel, followed by the bolometric luminosity in the middle, and the observed $B$-band luminosity in the bottom panel. While the bolometric luminosity peaks twice, the first peak is obscured, hiding much of the $\mathrm{BH}$ growth which is invisible in the optical. The quasar emerges from the obscuration, during a reddened phase (highlighted by the shaded stripe), eventually revealing the unobscured $\mathrm{BH}$ growth. 


\section{Conclusions}

We have identified a population of dust-obscured red quasars selected using a method that identified red-colored objects with detections in the radio and near-infrared. Based on their number counts compared to a well matched blue quasar comparison sample, we find that their fraction is $20 \%$ of the total quasar population, down to $K \leq 14.5$ magnitudes. Once we correct their observed brightness for extinction, we find that they are the most luminous sources at every redshift. High resolution imaging of a subsample of red quasar hosts reveals that they reside in highly disturbed, interacting galaxies. Many are accreting at or above the Eddington limit, and lie below the $M_{\mathrm{BH}}-$ $L_{\text {Bulge }}$ relation, suggesting that the black holes are rapidly growing to catch up to the relation for inactive galaxies. Our interpretation is that reddened quasars are revealing an emergent phase where the heavily obscured quasar is shedding its cocoon of dust prior to becoming a "normal"

blue quasar. Based on the fraction of objects in this phase, the duration of this cycle is $\sim 20 \%$ as long as the unobscured quasar phase: a few million years.

\section{References}

[1] Kormendy, J. 2004, Coevolution of Black Holes and Galaxies, 1

[2] Ferrarese, L., \& Merritt, D. 2000, ApJ, 539, L9

[3] Gebhardt, K., Bender, R., Bower, G., et al. 2000, ApJ, 539, L13

[4] Marconi, A., \& Hunt, L. K. 2003, ApJ, 589, L21

[5] Woo, J.-H., Treu, T., Barth, A. J., et al. 2010, ApJ, 716, 269

[6] Shaver, P. A., Wall, J. V., Kellermann, K. I., Jackson, C. A., \& Hawkins, M. R. S. 1996, Nature, 384, 439

[7] Boyle, B. J., \& Terlevich, R. J. 1998, MNRAS, 293, L49

[8] Wall, J. V., Jackson, C. A., Shaver, P. A., Hook, I. M., \& Kellermann, K. I. 2005, A\&A, 434, 133

[9] Sanders, D. B., Soifer, B. T., Elias, J. H., et al. 1988, ApJ, 325, 74

[10] Hopkins, P. F., Hernquist, L., Cox, T. J., et al. 2006, ApJS, 163, 1

[11] Di Matteo, T., Springel, V., \& Hernquist, L. 2005, Nature, 433, 604

[12] Dunlop, J. S., McLure, R. J., Kukula, M. J., et al. 2003, MNRAS, 340, 1095

[13] Floyd, D. J. E., Kukula, M. J., Dunlop, J. S., et al. 2004, MNRAS, 355, 196

[14] Floyd, D. J. E., Axon, D., Baum, S., et al. 2010, ApJ, 713, 66

[15] Schmidt, M. 1963, Nature, 197, 1040

[16] Greenstein, J. L. 1963, Nature, 197, 1041

[17] Sandage, A. 1965, ApJ, 141, 1560

[18] Glikman, E., Helfand, D. J., \& White, R. L. 2006, ApJ, 640, 579

[19] Urry, C. M., \& Padovani, P. 1995, PASP, 107, 803

[20] Zakamska, N. L., Strauss, M. A., Krolik, J. H., et al. 2003, AJ, 126, 2125 
[21] Zakamska, N. L., Strauss, M. A., Heckman, T. M., Ivezić, Ž., \& Krolik, J. H. 2004, AJ, 128, 1002

[22] Zakamska, N. L., Schmidt, G. D., Smith, P. S., et al. 2005, AJ, 129, 1212

[23] Zakamska, N. L., Strauss, M. A., Krolik, J. H., et al. 2006, AJ, 132, 1496

[24] Reyes, R., Zakamska, N. L., Strauss, M. A., et al. 2008, AJ, 136, 2373

[25] Lawrence, A. 1991, MNRAS, 252, 586

[26] Treister, E., Virani, S., Gawiser, E., et al. 2009, ApJ, 693, 1713

[27] Treister, E., Krolik, J. H., \& Dullemond, C. 2008, ApJ, 679, 140

[28] Warren, S. J., Hewett, P. C., \& Foltz, C. B. 2000, MNRAS, 312, 827

[29] Becker, R. H., White, R. L., \& Helfand, D. J. 1995, ApJ, 450, 559

[30] Glikman, E., Gregg, M. D., Lacy, M., et al. 2004, ApJ, 607, 60

[31] Glikman, E., Helfand, D. J., White, R. L., et al. 2007, ApJ, 667, 673

[32] Urrutia, T., Becker, R. H., White, R. L., et al. 2009, ApJ, 698, 1095

[33] Glikman, E., Urrutia, T., Lacy, M., et al. 2012, Submitted to ApJ

[34] Gordon, K. D., \& Clayton, G. C. 1998, ApJ, 500, 816

[35] White, R. L., Becker, R. H., Gregg, M. D., et al. 2000, ApJS, 126, 133

[36] Becker, R. H., White, R. L., Gregg, M. D., et al. 2001, ApJS, 135, 227

[37] York, D. G., Adelman, J., Anderson, J. E., Jr., et al. 2000, AJ, 120, 1579

[38] Schneider, D. P., Richards, G. T., Hall, P. B., et al. 2010, AJ, 139, 2360

[39] Urrutia, T., Lacy, M., \& Becker, R. H. 2008, ApJ, 674, 80

[40] UrrutiaT., Lacy, M., Spoon, H., Glikman, E., et al. 2012, Submitted to ApJ

[41] Porciani, C., Magliocchetti, M., \& Norberg, P. 2004, MNRAS, 355, 1010

[42] Hopkins, P. F., Hernquist, L., Martini, P., et al. 2005, ApJ, 625, L71 